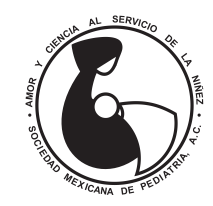

\title{
Impacto de la utilización de sistemas de medición de glucosa intersticial en el control glucémico en pacientes pediátricos con diabetes mellitus tipo 1
}

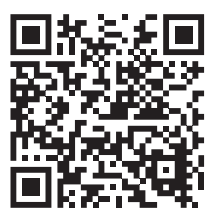

\author{
Sara María Barbed-Ferrández, ${ }^{\ddagger * *}$ Teresa Montaner-Gutiérrez, ${ }^{\S}$ Gemma Larramona-Ballarín,§ \\ Marta Ferrer-Lozano," Gracia María Lou-Francés\| \\ ‡ Médico Interno Residente de Pediatría. Hospital Universitario Miguel Servet; ${ }^{\S}$ Profesor Universidad de Zaragoza; \\ I| Unidad de Endocrinología y Diabetes Pediátrica, Hospital Universitario Miguel Servet, Zaragoza, España.
}

\begin{abstract}
RESUMEN
Introducción: El adecuado control glucémico en la diabetes mellitus tipo 1 (DM-1) es fundamental. Existen sistemas de medición de glucosa intersticial (MGI) que permiten realizar ajustes terapéuticos más específicos. El objetivo del estudio fue determinar el impacto de sistemas MGI en el control glucémico en pacientes menores de 18 años con DM-1. Material y métodos: Estudio de tipo cohorte retrospectiva. Los datos se recogieron de la historia clínica del paciente y de una encuesta realizada a los pacientes y sus padres o tutores. Resultados: De un total de 120 pacientes, 63 utilizaban un sistema MGI; después de seis meses de su uso se observó reducción significativa $(p<0.05)$ de las cifras de hemoglobina glucosilida, pasando de $7.5 \% \pm 0.6$ a $7.13 \% \pm 0.47$. Además, hubo disminución significativa de la variabilidad glucémica, del número de glucemias capilares y de la frecuencia de hipoglucemias leve y grave. Conclusiones: Los sistemas MGI ayudan a mejorar el control glucémico de los pacientes pediátricos con $\mathrm{DM}-1$.
\end{abstract}

Palabras clave: Diabetes mellitus tipo 1, control glucémico, sistemas de medición de glucosa intersticial, $\mathrm{HbA} 1 \mathrm{c}$, niños, adolescentes.

\begin{abstract}
Introduction: Adequate glycemic control in type-1 diabetes mellitus (DM) is essential. Interstitial glucose measurement (IGM) systems allow specific therapeutic adjustments. The aim of the study was to determine the impact of IGM systems in glycemic control in patients under 18 years with type- 1 DM. Material and methods: Retrospective cohort study. Data were collected from medical records of the patient, as well as a survey of patients and their parents or guardians. Results: Of a total of 120 patients, 63 used an IGM system; after six months of use, a significant reduction $(p<0.05)$ in the glycated hemoglobin levels was observed, going from $7.5 \%$ \pm 0.6 to $7.13 \% \pm 0.47$. In addition, there was a significant decrease in glycemic variability, in the number of capillary blood glucose levels and in the frequency of mild and severe hypoglycemia. Conclusions: The IGM systems help improve glycemic control in pediatric patients with type-1 DM.
\end{abstract}

Keywords: Type 1 diabetes mellitus, glycemic control, interstitial glucose measurement systems, $\mathrm{HbA1c}$, children, adolescents.

\footnotetext{
*Correspondencia: SMBF, sarabarbed@gmail.com

Conflicto de intereses: Los autores declaran que no tienen. Citar como: Barbed-Ferrández SM, Montaner-Gutiérrez T, Larramona-Ballarín G, Ferrer-Lozano M, Lou-Francés GM. Impacto de la utilización de sistemas de medición de glucosa intersticial en el control glucémico en pacientes pediátricos con diabetes mellitus tipo 1. Rev Mex Pediatr. 2020; 87(1):13-17. doi: 10.35366/93262 [Impact of the use of intersticial glucose sensors on glycemic control in pediatric patients with type 1 diabetes mellitus]
}

\section{INTRODUCCIÓN}

La diabetes mellitus tipo 1 (DM-1) es una de las enfermedades crónicas más frecuentes en la infancia, ocasionada por una deficiencia en la liberación de insulina debido a una destrucción de las células betapancreáticas productoras de esta hormona. ${ }^{1}$ El éxito en el manejo de esta enfermedad radica fundamentalmente en la obtención del control glucémico estricto para evitar las 
secuelas a largo plazo por hiperglucemia crónica, así como la hipoglucemia.

Por otra parte, es fundamental establecer objetivos realistas para cada niño y familia, capacitando a ambos para que brinden la atención diaria adecuada a fin de mantener un crecimiento, desarrollo y maduración emocional apropiados, así como facilitar la independencia del paciente para que lleve un autocuidado óptimo. ${ }^{1-3}$ Este autocuidado puede resultar difícil, ya que los perfiles glucémicos de un mismo paciente con frecuencia son irregulares, ${ }^{4,5}$ haciéndose necesaria la obtención de múltiples glucemias capilares diarias para realizar las modificaciones terapéuticas pertinentes.

Desde hace unos años existen dispositivos capaces de mostrar los niveles glucémicos en tiempo real o de forma retrospectiva que indican las variaciones glucémicas. También hay dispositivos combinados con bomba de insulina (sistema integrado) que suspenden automáticamente la infusión cuando se prevé una hipoglucemia, reiniciándose cuando la situación se resuelve. Mientras que los sistemas flash dan una lectura a demanda de la glucosa y del trazado de las últimas horas cuando se acerca el receptor al sensor. Con cualquiera de estos sistemas la medición de glucosa se basa en el líquido intersticial, y utilizan sensores mínimamente invasivos para la medición enzimática, ${ }^{4,5}$ por lo cual se denominan sistemas de medición de glucosa intersticial (sistemas MGI), o bien, sistemas de medición o monitorización continua de glucosa (sistemas MCG).

Se ha demostrado que los sistemas MGI optimizan el manejo de la DM-1, observando mejor control metabólico en pacientes motivados con su uso (especialmente adultos), prevención de hipoglucemias graves y reducción en la ansiedad que genera su aparición, además de mayor independencia para el manejo de la enfermedad. ${ }^{6}$ Sin embargo, no todo es perfecto, pues existen barreras financieras, físicas, psicológicas y tecnológicas que deberán tomarse en cuenta para asegurar el éxito. ${ }^{6}$ Además, en pacientes pediátricos existen pocos estudios al respecto.

El objetivo del estudio fue relacionar el impacto de la utilización de los dispositivos mencionados en el control glucémico en pacientes menores de 18 años con DM-1 atendidos en una unidad de diabetes pediátrica de un hospital de referencia.

\section{MATERIAL Y MÉTODOS}

\section{Diseño del estudio}

Estudio de tipo cohorte retrospectiva en el que se comparan dos grupos de pacientes. Uno de los grupos lo componen pacientes que utilizan sistemas de medición de glucosa intersticial para el manejo de la DM-1 durante seis meses previos al estudio y el segundo grupo son pacientes que no usan ninguno de estos sistemas durante los 12 meses previos al estudio o bien, nunca los han utilizado. En el momento del estudio, las familias adquirieron el sistema, ya que en España no está financiado por el Sistema Nacional de Salud.

El estudio se llevó a cabo durante el periodo de abril a diciembre de 2018. Los datos procedían de dos fuentes, por una parte, se diseñó un cuestionario que fue enviado por correo electrónico a los padres y/o tutores legales de los pacientes que aceptaron participar en el estudio, recogiendo los datos de forma prospectiva. Por otro lado, se revisaron los expedientes de los pacientes para registrar los datos de control glucémico en las visitas médicas habituales, tales como hemoglobina glucosilada y la presencia de hipoglucemias.

Previo a su realización, el proyecto de investigación fue revisado y aprobado por el comité ético de investigación clínica. Para su participación los padres o tutores legales firmaron carta de consentimiento informado; los pacientes mayores de 12 años firmaron consentimiento informado.

\section{Selección de los participantes}

La población de referencia procede de los pacientes atendidos en una unidad de diabetes pediátrica de referencia de una comunidad autónoma, cuyo inicio de la enfermedad fue entre los años 2003 y 2017. Dicha unidad está conformada por un equipo médico y de enfermería encargados del manejo integral del paciente, desde el momento del inicio de la enfermedad hasta los 18 años de edad. A partir del diagnóstico de DM-1, se da seguimiento a los pacientes cada uno, tres, seis o 12 meses de acuerdo con el tratamiento recibido y el control glucémico. Tanto pacientes como padres y/o cuidadores primarios reciben por igual clases de educación para el automanejo de la enfermedad.

\section{Variables de estudio}

Se incluyeron datos de sexo, edad al inicio, tiempo de evolución, variables de control glucémico (HbA1c, coeficiente de variabilidad glucémica, número de hipoglucemias, número de glucemias capilares diarias), tipo de tratamiento, tipo de sistema de medición de glucosa utilizado (sólo glucemias capilares diarias o uso del sistema MGI, considerando los tres tipos, sistema integrado, continuo e intermitente o flash). 
Las variables de control glucémico representan la media del año previo al estudio en el caso de pacientes que no usaban el sistema MGI. En aquellos pacientes que utilizaban sistemas MGI, las variables representan la media de los seis meses previos a su inicio y de los seis meses posteriores a su uso.

Análisis estadístico. Para la estadística descriptiva los datos se sintetizaron empleando medidas de tendencia central (promedio) y de dispersión (desviación típica o valores mínimo y máximo). En cuanto a la estadística inferencial, la comparación entre grupos se hizo con t de Student y con la prueba de $\chi^{2}$. El umbral de significación estadística fue $\mathrm{p}<0.05$. Los análisis se realizaron con el programa SPSS versión 23.0.

\section{RESULTADOS}

De 150 pacientes posibles, se incluyeron 120 pacientes (tasa de participación 80.5\%). Predominaron ligeramente pacientes del sexo masculino (56.7\%); al inicio de la enfermedad hubo pacientes de uno y hasta 15 años de edad, siendo el promedio de $6.7 \pm 3.8$ años. En el momento del estudio, el tiempo de evolución fue de $4.4 \pm 3.4$ años (desde uno y hasta 15 años).

De los 120 pacientes, poco más de la mitad utilizaban sistemas MGI ( $\mathrm{n}=63,52.5 \%)$. La comparación de las características entre el grupo con y sin uso de MGI se describen en la Tabla 1; en general, ambos grupos son similares, con excepción de que había un poco más de pacientes masculinos en el grupo con MGI y con menor tiempo de evolución de diabetes.

En el grupo con sistema MGI, a los seis meses de usar el dispositivo hubo una reducción significativa ( $p$ $<0.05$ ) de las cifras de HbA1c, de $7.5 \pm 0.6$ disminuyó a $7.13 \pm 0.47$. En referencia al coeficiente de variabilidad glucémica, el uso del sistema MGI demostró una disminución significativa de la variabilidad en $19 \%$. Cuando se analizó en pacientes que llevaron MGI en algún momento de su evolución y dejaron de usarlo, la variabilidad aumentaba en $12 \%$.

Por otro lado, con respecto al número de glucemias capilares al día en el grupo de pacientes con MGI, antes de su uso $84 \%$ de los pacientes realizaban entre tres y seis glucemias al día, en comparación con $43 \%$ tras seis meses de su uso.

En cuanto a la presencia de hipoglucemias, en la Tabla 2 se describen los porcentajes de hipoglucemias leves antes y después del uso de MGI. Se consideró a las hipoglucemias nivel $1(<70 \mathrm{mg} / \mathrm{dL})$ y a las hipoglucemias nivel $2(<54 \mathrm{mg} / \mathrm{dL})$ sin pérdida de conciencia ni sintomatología neurológica. Como se observa, hubo reducción significativa de hipoglucemias leves, en particular en los pacientes que tenían más de cinco episodios en dos semanas, pasando de $41.7 \%$ a $10 \%$ y $15 \%$, a los tres y seis meses, respectivamente. De las hipoglucemias graves, $7.5 \%$ había tenido al menos un episodio antes del uso del MGI, y no se registró ningún episodio durante los seis meses de uso.

\section{DISCUSIÓN}

Los sistemas MGI representan en la actualidad uno de los avances más innovadores en lo referente a tecnologías aplicadas al manejo y control de la DM-1, su uso en población pediátrica está aprobado en la actualidad. ${ }^{1}$ Datos recientes tanto del T1D Exchange Registry in the US como del Prospective DPV Diabetes Documentation and Quality Management System from Germany and Austria sugieren que el uso de los sistemas MGI en población pediátrica está creciendo de forma exponencial, el cual muestra un crecimiento mayor en niños preescolares y escolares. ${ }^{7,8}$ En nuestra muestra, más de la mitad de los pacientes son usuarios de algún sistema MGI, con predominio de los pacientes escolares y preescolares frente a los adolescentes.

Estudios a corto plazo han demostrado beneficios desde el punto de vista clínico con el uso de estos dispositivos, lo que permite un mejor control de la administración de insulina. ${ }^{9-12}$ El problema en lo referente a su uso en este grupo de población radica en la escasez de evidencia disponible en la actualidad, ya que un

\begin{tabular}{|c|c|c|}
\hline Variable & $\begin{array}{c}\text { Grupo sin } \\
\text { MGI }(n=57)\end{array}$ & $\begin{array}{l}\text { Grupo con } \\
\text { MGI }(n=63)\end{array}$ \\
\hline Edad al estudio (años) & $12.7 \pm 3.8$ & $9.79 \pm 4.14$ \\
\hline Edad al diagnóstico (años) & $7.1 \pm 3.8$ & $6.4 \pm 3.9$ \\
\hline \multicolumn{3}{|l|}{ Sexo, $n(\%)$} \\
\hline Masculino & $28(49.1)$ & $40(63.5)$ \\
\hline Femenino & $29(50.9)$ & $23(36.5)$ \\
\hline Tiempo de evolución (años) & $5.6 \pm 3.7$ & $3.3 \pm 2.8$ \\
\hline \multicolumn{3}{|l|}{ Tipo terapia insulina } \\
\hline Múltiple dosis insulina & $53(92.9)$ & $32(50.8)$ \\
\hline Bomba infusión subcutánea & $4(7.1)$ & $31(49.2)$ \\
\hline $\begin{array}{l}\text { Hemoglobina glucosilada } \\
\text { previo al estudio (\%) }\end{array}$ & $7.4 \pm 1.0$ & $7.5 \pm 0.6$ \\
\hline
\end{tabular}


Tabla 2: Porcentaje de pacientes con episodios

de hipoglucemia leve en dos semanas, antes

y después de iniciar el uso del sistemas

de medición de glucosa intersticial (MGI).

\begin{tabular}{cccc} 
& & \multicolumn{2}{c}{ Con uso de sistema MGI } \\
\cline { 3 - 4 } $\begin{array}{c}\text { Episodios de } \\
\text { hipoglucemia* }\end{array}$ & $\begin{array}{c}\text { Pre MG| } \\
(\%)\end{array}$ & $\begin{array}{c}\text { Tres meses } \\
(\%)\end{array}$ & $\begin{array}{c}\text { Seis meses } \\
(\%)\end{array}$ \\
\hline$<3$ & 21.6 & 50.8 & 56.6 \\
$3-5$ & 36.7 & 39.0 & 28.3 \\
$>5$ & 41.7 & 10.0 & 15.0 \\
\hline${ }^{*}$ * Medidos en dos semanas. ** Seis meses antes de iniciar uso de MGI.
\end{tabular}

alto porcentaje de los estudios publicados se centran en población adulta o adultos y niños conjuntamente. Uno de los estudios con mayor relevancia es el multicéntrico, controlado y aleatorizado que promovió la Juvenile Diabetes Research Foundation realizado en 322 pacientes adultos y niños con DM-1 bajo tratamiento insulínico intensivo, asignados al grupo control (glucemias capilares) o al grupo que utilizaba sistemas MGI. Este estudio mostró diferencias significativas en los niveles de $\mathrm{HbA1c}$ en pacientes $>25$ años, pero no en los menores de esta edad. ${ }^{13}$ No obstante, un análisis secundario demostró beneficios en todas las edades cuando el sensor se usó por más de seis días/ semana. ${ }^{14}$ En nuestro estudio sí encontramos una mejoría significativa en las cifras de HbA1c a los seis meses de uso del sistema MGI tanto en pacientes en tratamiento con MDI como con ISCI, probablemente porque casi todos los pacientes lo usaron todos los días de la semana.

Asimismo, los resultados de este estudio son similares a los de estudios realizados a partir del año 2010, con sistemas MGI de cuarta y quinta generación, los cuales demostraron consistentemente mejoría en el control glucémico en niños y en adultos, tanto por cifras de $\mathrm{HbA} 1 \mathrm{c}$ como por menor variabilidad glucémica, ${ }^{8}$ lo cual ocurrió sobre todo en pacientes que diario utilizaron el sistema. ${ }^{10,11,15,16}$

Por otra parte, un estudio aleatorizado de Battelino y colaboradores ${ }^{16}$ realizado con 153 pacientes, de los cuales 72 eran niños, demostró menor variabilidad glucémica en el grupo que utilizaba sistemas MGI y una reducción significativa en la cifra media de mediciones glucémicas capilares. Estos resultados concuerdan con los nuestros, dado que hubo reducción de la variabilidad glucémica y además, por incremento en esta variabilidad en quienes dejaron de utilizar el medidor. Del mismo modo, casi la mitad de los pacientes redujeron el número de glucemias capilares realizadas al día a los seis meses de su uso.

Con respecto a la presencia de hipoglucemias, estudios previos demostraron disminución significativa en la frecuencia de hipoglucemia leve y en la reducción del tiempo de hipoglucemia. ${ }^{8,17}$ En nuestro caso, demostramos reducción significativa del número de hipoglucemias leves diarias en pacientes que antes del uso del sistema tenían más de cinco hipoglucemias leves en dos semanas. Con las hipoglucemias graves existen resultados heterogéneos en los diversos estudios. ${ }^{8}$ Basados en nuestra experiencia, en los pacientes evaluados no hubo eventos hipoglucémicos graves.

Dado el crecimiento exponencial de estas herramientas para el manejo de la DM-1 en los últimos años, es preciso tomar en cuenta que uno de los factores asociados al éxito en su utilización es la capacitación en el uso de los sistemas MGI, sobre todo durante los primeros meses. ${ }^{8}$ De hecho, uno de los posibles motivos de los resultados favorables en este estudio puede ser la adhesión al uso del sistema, ya que pacientes y familias reciben capacitación y están motivados en su uso. Esto mismo se ha observado en otros estudios, como el estudio STAR $3,{ }^{17}$ que al comparar niños frente a adolescentes hubo mayor utilización del sensor en este primer grupo.

A pesar de los avances, todavía existen retos. Por ejemplo, casi la mitad de los pacientes del grupo control refería haber usado este tipo de sistemas en el pasado. Los motivos para no utilizarlos fueron su precio elevado, el rechazo por parte del paciente y la inseguridad en la medición del nivel glucémico. En efecto, se ha documentado que el coste es una de las cargas más negativas para el uso de estos sistemas; mientras que los pacientes adultos refieren sentirse preocupados por el gasto por su uso prolongado, los adolescentes sienten preocupación por perder un dispositivo tan costoso. ${ }^{18-20}$ Otro motivo del rechazo por parte del paciente es que el sensor puede ser difícil de ocultar.

\section{CONCLUSIONES}

La utilización de sistemas de medición de glucosa intersticial en pacientes menores de 18 años con DM-1 mejora el control glucémico. No obstante, son necesarios más estudios para determinar sus efectos a largo plazo. 


\section{REFERENCIAS}

1. Levitsky LL, Misra M. Management of type 1 diabetes mellitus in children and adolescents. Post TW, ed. UpToDate. Hoppin AG: UpToDate Inc. [Access August 2019] Available in: https://www. uptodate.com.

2. Chiang JL, Kirkman MS, Laffel LM, Peters AL. Type 1 diabetes through the life span: a position statement of the American Diabetes Association. Diabetes Care. 2014; 37: 2034.

3. American Diabetes Association. Children and Adolescents: Standards of Medical Care in Diabetes-2018. Position statements. Diabetes Care. 2018; 41 (Supplement 1): S126-S136. doi: 10.2337/ dc18-S012.

4. Torres LM, Barrio CR, García CB, Gómez GA, González Cl, Hermoso LF et al. Estado actual y recomendaciones sobre la utilización de los sistemas de monitorización continua de glucosa en niños y adolescentes con diabetes mellitus tipo 1. An Pediatr (Barc). 2011; 75(2): 134.e1-134.e6.

5. Giménez M, Diaz-Soto G, Andía V, Ruiz de Adana MS, GarcíaCuartero B, Rigla $\mathrm{M}$ et al. Documento de Consenso SED-SEEP sobre el uso de la MCG en España. Grupo de Trabajo de Tecnologías aplicadas a la Diabetes de la SED y de la SEEP. España. 2017.

6. Taddeo RL, Moser JT, Minnock PP. Continuous glucose monitoring in Pediatrics: the gap between potential benefits and the reality of utility. Pediatr Endocrinol Rev. 2017; 14 (Supplement 2): 435-440.

7. De Salvo D, Miller K, Hermann J, Maahs D, Hofer S, Clements $\mathrm{M}$ et al. Continuous Glucose Monitoring (CGM) and Glycemic Control Among Youth with Type 1 Diabetes (T1D): International comparison from the T1D Exchange (T1DX) and the DPV Initiative. 43rd Annual Conference of the International Society for Pediatric and Adolescent Diabetes; Innsbruck, Austria 2017.

8. Sherr JL, Tauschman M, Battelino T, De Bock M, Forlenza G, Roman $\mathrm{R}$ et al. ISPAD Clinical Practice Consensus Guidelines 2018: diabetes technologies. Pediatr Diabetes. 2018; 19 (Suppl 27): 302-325. doi: 10.1111/pedi.12731.

9. Battelino T, Phillip M, Bratina N, Nimri R, Oskarsson P, Bolinder $\mathrm{J}$. Effect on continuous glucose monitoring on hypoglycemia in type 1 diabetes. Diabetes Care. 2011; 34: 795-800.

10. Chase HP, Beck RW, Xing D, Tamborlane WV, Coffey J, Fox LA et al. Continuous glucose monitoring in youth with type 1 diabetes: 12-month follow-up of the Juvenile Diabetes Research Foundation continuous glucose monitoring randomized trial. Diabetes Technol Ther. 2010; 12(7): 507-515.
11. The Juvenile Diabetes Research Foundation Continuous Glucose Monitoring Study Group. Effectiveness of continuous glucose monitoring in a clinical care environment: evidence from the Juvenile Diabetes Research Foundation continuous glucose monitoring (JDRFCGM) trial. Diabetes Care. 2010: 33: $17-22$.

12. Ludvigsson J, Hanas R. Continuous subcutaneous glucose monitoring improved metabolic control in pediatric patients with type 1 diabetes: a controlled crossover study. Pediatrics. 2003; 111: 933-938.

13. Tamborlane WV, Beck RW, Bode BW, Buckingham B, Chase HP, Clemons $\mathrm{R}$ et al. The Juvenile Diabetes Research Foundation Continuous Glucose Monitoring Study Group. Continuous glucose monitoring and intensive treatment of type 1 diabetes. $N$ Engl J Med. 2008; 359: 1464-1476.

14. Beck RW, Buckingham B, Miller K, Wolpert H, Xing D, Block JM et al. Factors predictive of use and of benefit from continuous glucose monitoring in type 1 diabetes. Diabetes Care. 2009; 32(11): 19471953.

15. Pickup JC, Freeman SC, Sutton AJ. Glycaemic control in type 1 diabetes during real time continuous glucose monitoring compared with self monitoring of blood glucose: meta-analysis of randomised controlled trials using individual patient data. BMJ. 2011; 343: d3805.

16. Battelino T, Conget I, Olsen B, Schutz-Fuhrmann I, Hommel E, Hoogma $R$ et al. The use and efficacy of continuous glucose monitoring in type 1 diabetes treated with insulin pump therapy: a randomised controlled trial. Diabetologia. 2012; 55(12):3155-3162.

17. Bergenstal RM, Tamborlane WV, Ahmann A, Buse JB, Dailey G, Davis SN et al. Effectiveness of sensor-augmented insulin-pump therapy in type 1 diabetes. N Engl J Med. 2010; 363: 311-320.

18. Messer LH, Johnson R, Driscoll KA, Jones J. Systematic review or meta-analysis best friend or spy: a qualitative meta-synthesis on the impact of continuous glucose monitoring on life with type 1 diabetes. Diabet Med. 2018; 35(4): 409-418. doi: 10.1111/dme.13568.

19. James S, Perry L, Gallagher R, Lowe J. Diabetes educators: perceived experiences, supports and barriers to use of common diabetes-related technologies. J Diabetes Sci Technol. 2016; 10: 1115-1121.

20. Wysocki T, Hirschfeld F, Miller L, Izenberg N, Dowshen SA, Taylor A et al. Consideration of insulin pumps or continuous glucose monitors by adolescents with type 1 diabetes and their parents: stakeholder engagement in the design of web-based decision aids. Diabetes Educ. 2016; 42: 395-407. 